Hanno Merkt*

\title{
Begrüßung zum ZGR-Sondersymposium „Vom Konzern zum Einheitsunternehmen“
}

\author{
Liebe Kolleginnen und Kollegen,
}

im Namen aller ZGR-Herausgeber möchte ich Sie sehr herzlich zum ZGR-Sondersymposium „Vom Konzern zum Einheitsunternehmen“ begrüßen. Das Generalthema unseres Sondersymposiums müsste eigentlich mit einem großen Fragezeichen versehen sein. Ist es wirklich schon soweit? Ersetzt das Einheitsunternehmen europäischer Provenienz den Konzern, den wir als rechtskulturelle Errungenschaft erster Güte vornehmlich deutscher Herkunft kennen und schätzen, wie es uns Jan Thiessen in diesem Sammelband mit seinem einleitenden Grundlagenbeitrag im Rahmen der Max-Hachenburg-Gedächtnisvorlesung in Erinnerung ruft, war es doch Hachenburg, der sich bereits in der zweiten Hälfte der Weimarer Republik im HGB-Kommentar von Düringer/Hachenburg mit dem Kernproblem des Konzernrechts, der Abhängigkeit und ihrer rechtlichen Einhegung, insbesondere zum Schutz der Minderheitsaktionäre, auseinandersetzte?

Konzernrecht ist brandaktuell, das zeigen die seit längerem geführten Diskussionen um Lieferketten-Verantwortung ebenso wie die jüngsten Presseberichte über Internierungslager an chinesischen Standorten deutscher Tochtergesellschaften etwa von Volkswagen und BASF in der Provinz Xinjiang. Immer wieder geht es um die Frage: In welchem Maße sind deutsche Konzernmütter für ihre ausländischen Tochtergesellschaften verantwortlich? Während die einen darauf pochen, bei Fehlverhalten der Tochtergesellschaft stets den gesamten Unternehmensverbund in den Blick und auch in die Haftung zu nehmen, weil der Gesamtverband von der wirtschaftlichen Tätigkeit der Einzelgesellschaft profitiert, sehen die anderen den Trennungsgrundsatz, der eine zentrale ökonomische Funktion und Legitimation des Unternehmensverbunds ist, in Gefahr. Es besteht die reale Befürchtung, dass die im Kartell- und Wettbewerbsrecht entwickelte Theorie vom Einheitsunternehmen wie ein Flächenbrand auf andere Bereiche übergreift, nur als ein Beispiel genannt sei die internationale Zuständigkeit, die durch die Einheitsbetrachtung in ungeahnte Dimensionen geführt wird. Auch in anderen Bereichen erhöht der vertikale Durchgriff von den Töchtern auf die

\footnotetext{
* Der Autor ist Direktor des Instituts für Ausländisches und Internationales Privatrecht, Abt. II, an der Albert-Ludwigs-Universität Freiburg und Richter am Oberlandesgericht Karlsruhe.
} 
Mutter oder umgekehrt bzw. der horizontale Durchgriff die Risiken im Verbund ganz erheblich. Der Konzern als Organisationsform gerät unter gewaltigen Druck und könnte an Attraktivität verlieren. Und aus Berlin und Brüssel ist zu vernehmen, dass das Konzernrecht und insbesondere die Konzern-Compliance ganz oben auf der Agenda der deutschen EU-Ratspräsidentschaft im kommenden Jahr stehen werden. Die ZGR-Herausgeber denken, dass es angesichts dieser Aktualität des Konzernrechts dringend notwendig ist, der Frage nach der Zukunft des Konzernrechts nachzugehen, sich einerseits der Grundlagen des Konzernrechts zu vergewissern und andererseits seine Entwicklungsperspektiven auszuloten. Dazu haben wir vier Themenkomplexe zusammengestellt.

Erstens: Der Konzern als solcher, als Organisationsform aus der Praxis für die Praxis, steht nicht in Frage, auch wenn das Konzernrecht eine hoch kontroverse Materie ist und bleiben wird. Er ist als Organisationsstruktur aus dem modernen Wirtschaftsleben nicht wegzudenken, wenngleich sein Ansehen in der öffentlichen Wahrnehmung in jüngerer Zeit arg gelitten hat und etwa der Begriff „Bankenkonzerne“ ausgesprochen negativ besetzt ist. Welche ökonomische Legitimation und Leistungsfähigkeit hinter der Konzernstruktur steckt, soll uns der ökonomische Grundlagenbeitrag von Joachim Gassen von der Humboldt-Universität zu Berlin und Rolf Uwe Fülbier von der Universität Bayreuth noch einmal deutlich machen.

Zweitens: Die Entwicklung der Funktion des Konzernrechts hier in Deutschland verläuft in großen Wendungen. Angefangen als unkodifiziertes Organisationsrecht in der Vorkriegszeit, fortentwickelt nach dem Krieg vor allem als Recht zum Schutz vor dem Konzern, den der Gesetzgeber 1965 vor allem als Agglomeration mit qualifiziertem Schädigungspotential verstand, verbindet das Konzernrecht heute beide Ansätze zu einem schützenden Organisationsrecht. Diesem Funktionswandel wird der Beitrag von Andreas Engert von der Freien Universität Berlin nachgehen.

Drittens: Zentralthema gegenwärtig ist natürlich, es klang schon an, die Konzern-Compliance. Welche Pflichten treffen die Muttergesellschaft hinsichtlich ihrer Töchter? Ketzerisch gefragt: Sollte die Mutter auf Compliance in der Tochter verzichten, weil sie im Einheitsunternehmen auch ohne eigenes Verschulden für Verstöße der Tochter haftet? Oder gibt es Dämme, die durch angemessene Compliance-Maßnahmen der Mutter gehalten werden können? $\mathrm{Zu}$ diesem Themenkomplex werden wir Georg Seyfarth von Hengeler Mueller Düsseldorf hören.

Eng zusammen mit den Compliance-Pflichten hängen die zivilrechtlichen Einstandspflichten der Muttergesellschaft insbesondere in Lieferketten. Gerade dieses Thema hat, angefacht durch das Bekanntwerden von Missständen an ausländischen Produktionsstandorten, für große Aufmerksamkeit in der Öffentlichkeit gesorgt. Während viele Unternehmen erkannt haben, welche Bedeutung 
die Überwachung in der Lieferkette für ihre Reputation hat, sind die rechtlichen Dimensionen dieser Problematik noch nicht ansatzweise ausgeleuchtet. Dazu wird Marc-Philippe Weller von der Universität Heidelberg vortragen.

Und schließlich viertens: Besondere Brisanz erlangt im konzernrechtlichen Kontext natürlich das Kartellrecht, denn nirgendwo sonst wird das konzernrechtliche Trennungsprinzip mit vergleichbarer Schärfe und massiv getrieben von europäischen Vorgaben und EuGH-Rechtsprechung durch das Modell der wirtschaftlichen Einheit in Frage gestellt. Der wettbewerblichen Konzernhaftung und ihren Legitimationsgrundlagen wird sich Heike Schweizer von der HumboldtUniversität zu Berlin widmen, während das Konfliktfeld zwischen Wettbewerbsund Konzernrecht von Jens Prütting von der Bucerius Law School in Hamburg ausgeleuchtet wird. Soweit unser Programm.

Erlauben Sie mir schließlich noch, Herrn Kollegen Weller und seinem Team, allen voran Julia Rebecca Kohler, für die hervorragende Vorbereitung und Organisation dieses Symposiums sehr herzlich zu danken.

Und nun wünsche ich uns allen interessante Referate und anregende Diskussionen!

Hanno Merkt 
